Basabose, Philippe. "La représentation du livre dans

\title{
La représentation du livre dans Comment faire l'amour avec un Nègre sans se fatiguer de Dany Laferrière
}

\author{
Philippe Basabose \\ Memorial University \\ Canada
}

\section{Introduction}

La critique a vu dans le premier roman de Dany Laferrière, Comment faire l'amour avec un Nègre sans se fatiguer, divers projets d'écriture. Pour André Lamontagne, il s'agit d'un livre sur " l'altérité » (" 'On ne naît pas Nègre, on le devient' » 29). Lori Saint-Martin y a lu les " stéréotypes sexistes les plus éculés » (54), Charly Verstraet le « rapport du couple homme noir/femme blanche » (132). Jimmy Thibeault l'a étudié, avec L'énigme du retour (2010), un autre roman de Laferrière, comme " projet d'individualité " (24) et Aurélien Boivin comme " une dénonciation du racisme à travers la baise " (94). Sous sa dimension (auto)méta-critique, le roman lui-même semble orienter la critique vers certaines de ces pistes : "Gilles Marcotte parle de 'filtre de lucidité à travers lequel la violence et l'érotisme le plus cru acquièrent de la pureté' " (Laferrière, Comment faire l'amour avec un Nègre 153) ${ }^{1}$ ou encore : " Un professeur d'un collège de Montréal l'a recommandé à ses étudiants dans le cadre de son cours Racisme et société »(153). Ces repères critiques sont sans doute loin d'être exhaustifs et notre intention n'est pas forcément de les réfuter. Nous nous proposons seulement d'orienter la lecture de cette œuvre vers un angle que nous trouvons majeur mais qui semble avoir moins intéressé la critique, à savoir sa représentation du livre. Il s'agira donc d'appréhender l'objet-livre sous divers angles de sa phénoménologie tels qu'ils se déploient dans le roman.

En effet, le livre n'apparaît pas dans Comment faire l'amour avec un Nègre sans se fatiguer à titre de simples allusions mais comme un véritable motif structurant. L'univers du récit est peuplé de livres en tant qu'objets littéraires et d'objets-livres en tant que produits saisis dans leur processus de conception, d'élaboration, de distribution et de consommation ainsi que dans les effets que celle-ci peut susciter. II regorge aussi d'objets non-livres mais qui renvoient au livre et s'y rapportent à un degré ou à un autre comme les différentes rubriques de notre étude en rendront compte. Même la configuration topographique et l'architecture actancielle sont organisées dans cet esprit. Aussi, lire ce roman s'impose-t-il comme lire avec les livres mais aussi lire sur les livres. Vieux, le narrateur et personnage principal, garde, manipule, lit, écrit, commente des livres. II est, comme sa demeure et les lieux qu'il fréquente, un musée à livres. C'est de ce fil essentiel du roman que la présente étude détaille la texture en quatre temps : lieux du livre, rapport des personnages aux objets-livres, atelier du livre, effets du livre.

\section{Des lieux du livre}

Le cadre spatial du roman Comment faire l'amour avec un Nègre sans se fatiguer est la ville de Montréal. Si cette dernière est le théâtre global où les intrigues qui constituent le récit se déroulent, le foyer principal en est le domicile des deux principaux protagonistes. Le roman fournit force détails qui amènent le lecteur à connaître de ce lieu domiciliaire l'état, la chambrée, tant régulière que circonstancielle, le moindre des objets qui le meublent ainsi que leurs usages respectifs. Le fouillis pour le moins inextricable de ce réduit où corps et objets se mêlent jusqu'à l'entrechoquement trahirait-il seulement le désordre, le laisser-aller et les manières débraillées des personnages qui semblent faire la règle des lieux ? C'est ce qu'un œil qui se soucie plus des détails que du lien qui les soude ensemble pourrait être amené à croire. Et pourtant, pour peu que l'on examine le rapport qui unit chacun de ces détails à l'objet de valeur - au sens sémiotique du concept - qui circule dans le texte et le structure, lui donnant ainsi justification, à savoir le livre, on se rend à l'évidence que derrière le désordre apparent se trouve l'intention bien consciente d'écrire un livre sur le livre.

Le domicile élu pour abriter le livre comme objet de valeur du récit et l'acte d'écriture à travers lequel il naît est sis « au 3670, rue Saint-Denis, en face de la rue Cherrier » (11). Des précisions évaluatives de la chambre qui sert de foyer au récit sont fournies dès le début du récit : "C'est un abject un et demie [sic] que le concierge a refilé à Bouba pour un deux et demie [sic] à 120 dollars par mois » (11). Le narrateur ajoute : "On loge au troisième. Une chambre exiguë, coupée en deux par un affreux paravent japonais à grands oiseaux stylisés » 
Basabose, Philippe. " La représentation du livre dans Comment faire l'amour avec un Nègre sans se fatiguer de Dany Laferrière » Nouvelle Revue Synergies Canada, N 13 (2020)

(11). Quelques paragraphes plus bas, il qualifie la chambre de "bauge étroite » (12). Ce procès de péjorativisation - une bauge étant un lieu très sale, un taudis - participe à l'encodage du livre dans le fil du récit. D'une part, le narrateur se distancie quelque peu des énoncés descriptifs et évaluatifs du lieu pour en faire partager la responsabilité avec la machine comme objet écrivant - nous reviendrons au rôle que celui-ci joue dans la narration - : " UNE CHRONIQUE DE MA CHAMBRE AU 3670, RUE SAINT-DENIS (description faite avec l'accord de ma vieille Remington 22) »(106). D'autre part, l'atmosphère et la qualité du lieu sont données comme faisant partie de l'étoffe dont le texte est tissé car le narrateur établit un lien de corrélation entre son génie et l'étrangeté de l'aura dans laquelle le labeur - dans tous les sens du mot - de l'écriture baigne :

La chambre tangue légèrement sous l'effet Molson. Une épaisse chaleur entre, par vagues successives, dans mon dos. Les consonnes n'arrêtent pas de forniquer et d'engendrer, là, sous mon nez. La vaisselle traîne. La poubelle déborde. J'étouffe. Je regarde, sans force, les cafards vaquer à leurs occupations quotidiennes. La chambre baigne dans un jus ultramarin. Comment ne pas se prendre pour un génie dans de telles conditions $?^{2}(148)$

Quant à l'organisation et à l'ameublement de la pièce, des axes principaux peuvent être dégagés. Pour ce qui est des biens meubles, la pièce apparaît aussi pauvre que ses occupants. Elle est dominée par un divan, mot disséminé très abondamment dans le texte et chaque fois noté avec une majuscule, soit à l'initiale, soit dans son intégralité. L'objet est investi de deux fonctions principales. D'un côté, c'est le lieu de la lecture : « [...] Bouba s'est installé sur ce Divan avec la collection de l'œuvre de Freud, un vieux dictionnaire dont les premières lettres (ABCD et une partie de $E$ ) manquent et un volume dépenaillé du Coran » (12).

Que le chapitre liminaire du roman présente un des deux personnages principaux, Bouba, à l'œuvre de l'activité de la lecture, c'est là une façon de donner le ton à la narration. Comment faire l'amour avec un Nègre sans se fatiguer est en effet peuplé de livres choisis de la culture littéraire des personnages, et leur présence passe par diverses pratiques transtextuelles - dans toute la richesse et l'ouverture du concept de Gérard Genette - qui définit la transtextualité comme « tout ce qui met un texte en relation, manifeste ou secrète, avec un autre texte » (7) : citations, commentaires, simples allusions ou évocations, etc. Seul un décompte chiffré des œuvres qui ne pourrait aucunement prétendre à l'exhaustivité - car évoquer un auteur seulement peut signifier évoquer beaucoup d'œuvres - prouverait l'immensité du champ transtextuel du roman. Nous ne ferons pas ici d'étude détaillée de la dimension transtextuelle dans Comment faire l'amour avec un Nègre sans se fatiguer. Le volet est trop important pour être étudié de façon approfondie en parallèle avec un autre dans l'espace d'un article. II mériterait une étude à part. Signalons en passant que dans son essai intitulé Le roman québécois contemporain: les voix sous les mots (2004), André Lamontagne a consacré un chapitre à l'intertextualité dans ce roman de Dany Laferrière pour démontrer sa fonction dans la constitution identitaire du sujet noir dans ses rapports avec le sujet blanc.

Ailleurs dans le texte, à la lecture sur le divan s'adjoignent l'écoute de la musique - " Je vois ce vieux Divan où Bouba lit Freud en écoutant du jazz à longueur de journée » (108), la dégustation d'un thé ou les deux à la fois : "Charlie Parker crève la nuit. [...] Bouba est affalé sur le Divan dans sa pose habituelle à siroter du thé de Shanghai tout en feuilletant un bouquin de Freud »(13). De telles activités se doublent de consommation d'alcool et/ou de sexe dont nous évoquerons la place dans l'économie globale du texte et dans le projet d'écriture de Laferrière plus loin dans notre analyse. La valeur emphatique de la reprise presque intégrale de ce motif du divan et des actes dont il sert de siège dans les deux citations qui précèdent est elle-même assez parlante.

D'un autre côté, le divan sert de siège à la pratique psychanalytique déjà suggérée dans l'évocation de la figure du père de la psychanalyse. C'est dans le chapitre XII du roman intitulé " Miz Suicide sur le Divan » et qui s'ouvre ainsi : "Bouba est assis sur le Divan comme un vieux bhikku déchiffrant les idéogrammes de Li Po, avec Miz Suicide à ses pieds buvant chacune de ses paroles » (67). En plus de nommer l'analysant et l'analysée, le chapitre détaille le rapport qui les lie : "Bouba est son conseiller en matière de suicide. Elle ne s'intéresse à rien d'autre. Personne ne s'intéresse aussi à elle, à part Bouba qui la reçoit chaque mardi et chaque jeudi, de $16 \mathrm{~h}$ à $16 \mathrm{~h} 45$, ce qui fait trois thés de quinze minutes chacun » (67).

Il serait intéressant ici de noter le type de cure auquel Bouba astreint sa patiente. Plus qu'un simple élément du décor, le rituel de la dégustation du thé fonctionne comme un prétexte pour inscrire les textes lus dans le roman en train de s'écrire. Le narrateur informe : «ll lit, avec sa voix gutturale et mystique, le précieux petit livre du poète à barbiche, Li Po, sur la manière de boire le thé » (67-68). De ce prétexte, la scène fait intervenir 
Basabose, Philippe. " La représentation du livre dans Comment faire l'amour avec un Nègre sans se fatiguer de Dany Laferrière » Nouvelle Revue Synergies Canada, N 13 (2020)

Vieux, le personnage narrateur qui fait figure de double de l'auteur. II réoriente le propos, l'écriture relecture, vers un autre nom qui cadre mieux avec le souci de la patiente : « Connais-tu Papini ? [...] Papini [...] a très intelligemment écrit sur le suicide » (69). Le propos précède et explique sa pertinence par rapport à la suite : "Miz Suicide ne s'intéresse qu'aux sujets qui traitent de la mort » (69). De là, Vieux ouvre une brèche littéraire qui lui permet de présenter l'auteur : " Papini est un écrivain italien, un homme fortement désabusé. Dans l'un de ses livres, il raconte I'histoire d'un Allemand qui cherche à se suicider » (70). Miz Suicide souffrirait, comme on s'en aperçoit à travers cet extrait, de l'angoisse de la mort, et non comme un sujet de répulsion mais d'attrait, le problème résidant dans la façon : « [...] Cet homme doux et civilisé cherche un moyen courtois pour se suicider [...] toutes les manières [...] lui paraissent brutales, stupides ou vulgaires » (70).

Le divan comme siège de la co-événementialité et de la complémentarité des pratiques de la psychanalyse et de la lecture refait surface dans le chapitre XIV intitulé "Comme une fleur au bout de ma pine nègre ». Quoique le chapitre épouse, dans l'enchaînement de ses actions, le propos de son titre et pourrait servir de chapitre emblématique du thème de la sexualité pour tout le roman, il se termine sur une scène qui replace le narrateur dans la dimension clé de l'œuvre, à savoir la littérature et son métonyme, le livre. Vieux raconte : " Je m'installe sur le Divan avec un bouquin de Borges et, trente secondes plus tard, les premières notes de Take The A Train remplissent la pièce » (87). Que Borges côtoie Papini dans le même texte de Laferrière dont le personnage narrateur a toutes les caractéristiques du double de l'auteur, comme déjà souligné, voilà qui écarte la part du hasard et explique tout. De Vieux lecteur de Papini et de Borges à l'auteur, Laferrière, il n'y a d'écart que de l'ordre de l'instance narrative. Autrement, le rapport entre les trois noms se démontre clairement. Dans sa préface au Miroir qui fuit (2006) (signé Giovanni Papini), Jorge Luis Borges écrit :

J'ai lu Panini et je l'ai oublié. Sans m'en douter, je me comportais de la manière la plus sagace - peutêtre l'oubli est-il une forme profonde de la mémoire. Quoi qu'il en soit, je veux rapporter une expérience personnelle. À présent, en relisant ces pages si lointaines, je découvre en elles, avec reconnaissance et stupéfaction, des fables que j'avais cru inventer et que j'ai élaborées de nouveau à ma façon en d'autres circonstances de l'espace et du temps. Plus importante encore, la découverte d'un climat en tout point identique à celui de mes fictions. (Papini 9)

Et pour peu qu'on ait lu ou écouté Laferrière, on sait la place qu'occupe Borges dans son univers mental de lecteur et d'auteur. Le discours de sa réception à l'Académie Française compte à lui seul dix occurrences du nom du géant littéraire argentin ${ }^{3}$. Aussi, Alain Lessard, dans son entrevue avec Laferrière publiée par le magazine Nuit blanche, confie: "Pour Dany Laferrière, Borges égale plaisir, plaisir de lecture. Ainsi qu'élégance d'esprit. [...] À l'évidence, Dany Laferrière, avec ses formules aussi spontanées que débordantes de sens et d'images, n'a rien à envier au vieux maître Argentin ! » (34). Laferrière avoue, par cette occasion, avoir abordé Borges par son célèbre recueil de nouvelles, Fictions, et dans le même lieu où son narrateur accouche de son premier roman, c'est-à-dire, sur la rue Saint-Denis. À la question « Tous les lecteurs de Borges que je connais, moi y compris, ont abordé Borges par Fictions. Est-ce votre cas ? ", Laferrière a répondu : " Oui, moi aussi. J'étais sur la rue Saint-Denis et je suis entré dans une toute petite librairie [...]. J'a été fortement impressionné et je me suis dit que pour la première fois, il y avait quelque chose de nouveau, en littérature »(Lessard 34).

Salle de lecture et d'écriture, la chambre du 3670 rue Saint-Denis est aussi portraiturée comme un lieu d'entreposage de livres. Du chapitre XVIII du roman, intitulé « Une chronique de ma chambre au 3670, rue Saint-Denis ", et suivant la rhétorique de l'accumulation que le roman cultive à volonté, faisant alterner les segments verbaux "J'écris ", " Je vois ", " Je pense ", " J'écoute " " Je rêve " que complètent de longues séries de noms d'objets qui meublent la chambre ou qui passent par l'esprit du personnage affairé à taper sur sa machine à écrire, on peut lire :

J'écris: CAISSE DE BOUQUINS. Je lis: Hemingway, Miller, Cendrars, Bukowski, Freud, Proust, Cervantès, Borges, Cortázar, Dos Passos, Mishima, Apollinaire, Ducharme, Cohen, Villon, Lévy Beaulieu, Fennario, Himes, Baldwin, Wright, Pavese, Aquin, Quevedo, Ousmane, J.-S. Alexis, Roumain, G. Roy, De Quincy, Marquez, Jong, Alejo Carpentier, Atwood, Asturias, Amado, Fuentes, Kerouac, Corso, Handke, Limonov, Yourcenar. (108)

Dans cette série, c'est le monde entier qui est convié. Quel continent n'est pas en effet représenté par un ou plusieurs de ses auteurs ? II appert d'un examen plus proche des auteurs listés - il suffit que le lecteur s'attelle à l'exercice de mettre une époque et un lieu devant chaque nom -, que Comment faire l'amour avec un Nègre 
Basabose, Philippe. « La représentation du livre dans Comment faire l'amour avec un Nègre sans se fatiguer de Dany Laferrière » Nouvelle Revue Synergies Canada, Nº 13 (2020)

sans se fatiguer est une invitation au voyage à travers la littérature de tous les temps, quoique la saveur dominante reste moderniste, et de tous les horizons.

Autant les livres abondent dans le roman, autant tous les lieux sont bons pour s'accommoder à leur présence. Dans les lignes qui précèdent, nous avons privilégié le gros plan de la chambre comme espace global. Un cadrage plus réduit aboutit au même constat : la présence du livre. Que Bouba s'écarte de son divan ou Vieux de la table sur laquelle trône sa vieille Remington 22, l'objet-livre les accompagne partout dans la chambre : lit, toilettes, salle de bain, cuisine.

L'omniprésence du livre déborde le cadre focal du récit pour irradier vers tous les espaces convoqués dans ce dernier. Ces espaces peuvent être des lieux habituellement commis à la production et à la circulation du livre. C'est à ce titre que sont inscrits dans le texte la Librairie Hachette (54), la Bibliothèque nationale (67), le Palais du livre (77), les bibliothèques personnelles, notamment celles des chambres de Miz Littérature (102) ou de Miz Chat :

La plus petite pièce, qui a l'air de servir de boudoir, possède un divan jaune et une minuscule bibliothèque composée exclusivement de livres érotiques : la fameuse collection de J. J. Pauvert, les œuvres de Miller (Sexus, Plexus, Nexus), Histoire d'O, les livres édités par Régine Desforges, l'CEuvre amoureuse de Lucien de Samosate, l'Aretin [sic], Rachilde, Octave Mirbeau. (133-134)

C'est également dans ce genre de lieux publics de livres qui favorisent les rencontres sociales que les personnages qui circulent dans le roman ont fait connaissance. Vieux a rencontré Miz Littérature à une soirée littéraire à l'Université McGill (26) et Bouba a « repêché » (67) Miz Suicide " à la Librairie ésotérique, sur Saint-Denis, en face de la Bibliothèque nationale " (67). D'autres lieux plutôt atypiques pour la lecture abondent dans le texte : le bureau de poste, la rue, la terrasse, la cinémathèque, le parc, etc. Le postulat de l'omniprésence du livre est confirmé par un autre passage du texte qui va bien au-delà des espaces physiques ici évoqués et qui établit un rapport de convenance de l'activité de la lecture entre les éléments de l'objet-livre et du lieu de lecture. Et le rapport est établi sous un mode prescriptif :

Faut lire Hemingway debout, Basho en marchant, Proust dans un bain, Cervantès à l'hôpital, Simenon dans le train (Canadian Pacific), Dante au paradis, Dosto en enfer, Miller dans un bar enfumé avec hot dogs, frites et coke... Je lisais Mishima avec une bouteille de vin bon marché au pied du lit, complètement épuisé, et une fille à côté, sous la douche. (21)

Ce qu'il faut conclure de cette partie est que la topographie du roman Comment faire l'amour avec Nègre sans se fatiguer est rigoureusement moulée dans et sur l'objet-livre et que chaque lieu de l'intrigue se ramène d'une façon ou d'une autre à ce dernier et contribue à son destin. Le livre ne se concevrait, dans la logique laferriérienne véhiculée par ce récit, que par rapport à un lieu.

\section{Des personnages et des livres}

Le rapport de type implicatif qui unit l'actant objet du livre à l'actant humain du personnage perce déjà des pages précédentes de notre étude. Ce rapport vaut la peine d'être exploré à part plus en détails cependant. Ceci pour faire ressortir davantage l'une des facettes les plus élidées du premier roman de Laferrière mais pourtant la plus pertinente, il nous semble, à savoir la représentation du livre.

Notons d'abord que malgré le pointillisme des descriptions de ses personnages, des actions dans lesquelles ils sont engagés, des cadres qui abritent ces dernières et les éléments du décor choisis, le roman reste presque totalement muet sur les parcours des personnages. De ceux-ci, surtout Vieux et Bouba, ainsi que, mais dans une moindre mesure, les filles qui les fréquentent, le lecteur n'apprend que les goûts intellectuels, littéraires plus précisément, les soifs qui les mènent, les angoisses qui les hantent. Aucune manière de dresser un curriculum vitae pour les personnages principaux. Aucune possibilité non plus d'établir une fiche identitaire complète. La seule séquence de présentation reste non seulement partielle, mais aussi elle se passe de détails : "Bouba me présente. - Mon frère. On habite ensemble » (128), segment suivi d'une interrogation qui cherche à pousser plus loin dans le détail mais qui se heurte au même laconisme identificatoire : " Qu'est-ce que tu fais? me demande l'une d'elles. - J'écris. Écrivain. - Ah! bon. Qu'est-ce que tu écris ? - Des phantasmes. - Quel genre ? - Les miens. - Ça vaut la peine ? - On verra » (128-129). Aux identités, le roman préfère les typologies, la négritude (pour Vieux et Bouba) versus la blanchitude (pour les filles qui les fréquentent et alimentent leurs désirs), l'occidentalité qui correspond à la situation présente des personnages 
Basabose, Philippe. " La représentation du livre dans Comment faire l'amour avec un Nègre sans se fatiguer de Dany Laferrière » Nouvelle Revue Synergies Canada, N 13 (2020)

contre d'éventuelles origines étrangères ${ }^{4}$. Si la variable de l'ascendance et des origines est textuellement évoquée, c'est seulement pour être écartée de l'équation identitaire. Aussi, au sujet de l'absence de l' " appel à l'Afrique » (154) dans le roman que Vieux vient de sortir - du moins dans son rêve jusque-là -, l'explication est-elle sans détour :

Q. : [...] les Noirs font appel à l'Afrique dans ces cas-là. Vos personnages, non. Pourquoi ? R. : Parce que ce sont des Occidentaux. Q. : Ils sont musulmans! R. : Oui. Leur foi appartient à l'Islam, mais leur culture est totalement occidentale, si vous voulez : Allah est grand, mais Freud est leur prophète. $(154-155)$

La fin de non-recevoir opposée à la considération des origines avait été plus catégorique encore à une autre occasion. Commentant la question qu'une fille en compagnie de Miz Littérature lui avait posée, à savoir « Tu viens d'où ? [...] tu viens de quel pays ? " (112), Vieux avait lâché : "À chaque fois qu'on me pose ce genre de question, comme ça, sans prévenir, sans qu'il en ait été question, auparavant, du National Geographic, je sens monter en moi un irrésistible désir de meurtre »(112). Et pour couper court à la conversation, il donne une clarification qui brouille tout : "Le jeudi soir, je viens de Madagascar » (112). Fidèle à son personnage, Laferrière continuera à cultiver l'énigme en matière de l'être abusivement réduit à son lieu de provenance en signant, presqu'une vingtaine d'année après, un autre roman au titre pas moins énigmatique, pas moins provocateur, Je suis un écrivain japonais qui, lui aussi, met en garde contre "le piège de la pureté identitaire » (Je suis un écrivain japonais 263).

Une telle présentation des personnages en présence ou du point de vue de leurs projets plutôt que dans leur passé et leurs origines permet non seulement de refuser la fixité identitaire mais aussi de ne pas perdre de vue l'essentiel de l'objet de l'écriture, le livre. Et dans cet esprit, fixons quelques repères.

En dépit des actions secondaires qui contribuent à l'intrigue du roman et en accroissent la complexité, le récit de Comment faire l'amour avec un Nègre sans se fatiguer obéit à une unité d'action parfaite. Tout part et ramène à l'écriture d'un roman et le narrateur ne se définit dans sa réalité et dans ses rêves que par et par rapport à son projet d'écrire. L'écrivain à l'œuvre est aussi peint comme un dévoreur de livres. Pas de chapitre, voire pas de page, qui ne présente ou ne réfère, soit à un titre de livre, un auteur, un commentaire sur ce qui, de près ou de loin, renvoie à l'art d'écrire. La duplicité et la complicité auteur narrateur sont elles-mêmes métainscrites ${ }^{5}$ dans le texte, bien qu'a contrario, par l'illusion référentielle que le roman entretient. Cela se joue « à Radio Canada, dans la salle d'enregistrement de l'émission Noir sur Blanc » (152). À la journaliste, Miz Bombardier, qui l'interroge sur la véracité des faits racontés - « Est-ce vrai ? [...] Je vous demande ça parce que, dans la réalité, vous habitez encore au même endroit, au carré Saint-Louis, vous avez un ami chez vous et vous êtes écrivain comme votre narrateur » (154), - la réponse est : " Ce n'est que pure coïncidence » (154).

Bouba, l'ami et colocataire de Vieux, est aussi un lecteur assidu. Comme Vieux, il conjugue lecture et cogitations littéraires, beuverie, baise - pour faire écho au registre familier, vulgaire, qui empreint le roman -, réflexions sur les religions. II ne cesse non plus d'encourager Vieux, de s'enquérir et de lui prodiguer conseils et avertissements concernant son projet d'écriture. En témoignent ces deux passages : «Qu'est-ce qui t'arrive, Vieux ? [...] T'as peur de la maudite page blanche ? - C'est ça. - Tords-la, Vieux, prends-la, fais-la gémir, humanise cette saloperie de page blanche »(105-106) et «Réveille-toi, Vieux, ça fait une heure que tu dors sur la machine, sinon tu vas attraper un torticolis » (107). La personnification, la féminisation et l'érotisation de l'image métonymique du livre ou du processus qui aboutit au livre, à savoir la page - fût-elle blanche -, concourent toutes à une inscription plus prononcée du fragment dans le cadre du livre qui tient le récit. Bref, la construction du personnage de Bouba en fait un fidèle compagnon de route de Vieux et, par là, un maillon important dans la chaîne qui lie le roman à la thématique de l'art littéraire.

Dans l'entourage immédiat de Vieux se trouve Miz Littérature comme compagne et comme l'autre du couple Noir-Blanche. Prouver la conformité de celle qui porte le nom de Miz Littérature au schéma du motif du livre que nous avons pris pour le fil clé qui tient ensemble le texte peut paraître tautologique. Comme le récit luimême ne s'y soustrait pas mais bien au contraire, ici aussi faisons parler le texte. Miz Littérature est d'ailleurs le seul personnage dont l'identité est amplement détaillée, façon de mieux la poser et de l'imposer comme figure de l'altérité qui permet le rapprochement entre tous ceux que les défis et les atouts ou les avantages du vivre ensemble peuvent séparer ou réunir. 
Basabose, Philippe. " La représentation du livre dans Comment faire l'amour avec un Nègre sans se fatiguer de Dany Laferrière » Nouvelle Revue Synergies Canada, N 13 (2020)

Dès le quatrième chapitre, le lecteur apprend : « Miz Littérature [...] C'est la fille de l'Université McGill et c'est Bouba qui l'a surnommée Miz Littérature. C'est comme ça avec Bouba » (25). En lui donnant le nom de Miz Littérature, Bouba, personnage qui est si proche du narrateur et qu'on prendrait pour son double, la crée à son image d'amant de livres. La déclinaison de l'identité continue jusque sous l'angle familial mais là encore le pont avec la littérature ne flanche pas :

Miz Littérature a une famille importante, un avenir, de la vertu, une solide culture, une connaissance exacte de la poésie élisabéthaine et même, elle est membre d'un club littéraire féministe à McGill - les Sorcières de McGill - dont les membres s'occupent de remettre en circulation les poétesses injustement oubliées. Cette année, elles publient en édition de luxe, avec des encres de Valérie Miller, l'œuvre poétique d'Emily Dickinson. [...] Miz Littérature prépare sa thèse de doctorat sur Christine de Pisan. (42)

De plus, comme déjà indiqué, elle entretient dans sa chambre une petite bibliothèque et participe à des soirées littéraires dans la prestigieuse université de McGill. Comme ses hôtes, elle s'enivre de boisson et de poésie : " Joue contre joue. ... elles [Miz Littérature et Valérie Miller] hurlent (en chœur) le poème NON SYLVIA PLATH N'EST PAS MORTE. Miz Littérature s'arrête pour boire un peu avant de continuer. Elle se verse une bonne rasade de vin qu'elle avale d'un coup sec avant de reprendre le poème » (75).

Deux des autres Miz du roman procèdent, peu ou prou, de la même logique de l'adéquation des intérêts des personnages au projet formulé et poursuivi par le narrateur. Nous avons déjà souligné le cas de Miz Suicide qui attend de la littérature le soulagement de ses angoisses. La nommée Miz Sophisticated est également située dans le même sillage des amants du livre. Le narrateur informe à son sujet : "Une demi-heure après le repas, je l'ai vue sortir, subrepticement, un petit livre en cuir brun de son sac gucci. - C'est le livre de Mao ? Non. Je parie, dis-je, que c'est un livre de prières orientales » (77).

La synopsis des personnages définis à l'aune des fonctions qu'ils occupent dans les différentes séquences narratives du texte et de leur goût pour les livres pourrait se compléter par l'ajout de toutes les autres catégories de protagonistes disséminés sur la chaîne de la création et de la circulation de ce bien symbolique. II s'agit des critiques, des éditeurs, des libraires, des journalistes etc., qui, eux aussi, sont impliqués à divers degrés dans la narration. Nous ferons l'économie de cette amplification.

\section{De l'atelier du livre}

En mettant en scène un écrivain à l'œuvre, un écrivant (Hertel 7) en somme - la substantivation hertélienne ne tomberait jamais mieux à propos -, qui se construit comme auteur au fil du récit et finit en effet par l'être à son dénouement, Laferrière donne au lecteur l'occasion de pénétrer non seulement dans l'âme de l'écrivain mais aussi dans l'atelier de l'écriture. Les outils, les difficultés, les attitudes, les aides éventuelles, comme les aides impossibles, tout est décliné avec minutie.

Le premier allié de taille aux côtés du narrateur est sa machine à écrire, une " vieille Remington 22 »(53). L'outil n'a pas été choisi au hasard car, au dire de Vieux, il en est des livres comme des fils : telle machine, telle œuvre. Le roman abonde en mises en scène qui s'appesantissent sur le rôle de la machine. La Remington 22 acquiert sa renommée et son efficacité des célèbres mains qui l'ont utilisée par le passé. Le narrateur raconte: «Je m'installe devant la machine à écrire avec l'espoir de tirer quelque chose d'une Remington 22 qui a bien vu Joan Baez en chair et en os » (59) et précise :

Je l'ai achetée chez un brocanteur de la rue Ontario qui vend des machines à écrire avec pédigree. De vieilles machines. II les vend à de jeunes écrivains car qui d'autre qu'un jeune écrivain serait assez gogo pour croire à un truc si vulgairement commercial. Et qui d'autre aussi se croirait écrivain parce qu'il possède une machine ayant appartenu à Chester Himes, James Baldwin ou Henry Miller. $(59-60)$

Si le texte ne s'en tenait qu'à cela, le lecteur retiendrait la réserve du pis-aller du jeune écrivain qui, faute de moyens, se rabattrait sur des vieilleries. La suite montre bien la magie que Vieux attache à la machine : "Alors, lui, il vend des machines selon le style du bouquin que vous voulez écrire. Si c'est un bouquin paranoïaque, on vous vend la machine schizophrène qui a appartenu à Tennessee Williams; si vous voulez plutôt une machine suicidaire, il y a celle de Mishima » (59-60). Le paragraphe bien fourni continue l'inventaire mariant les choix d'écriture aux choix des machines. 
Basabose, Philippe. " La représentation du livre dans

Comment faire l'amour avec un Nègre sans se fatiguer de Dany Laferrière » Nouvelle Revue Synergies Canada, N 13 (2020)

La machine par ailleurs personnalisée est présentée comme une source d'où jaillissent les idées que la main qui tape couche sur le papier. "Je suis devant la machine depuis dix minutes, fait noter le narrateur, et j'essaie de soutirer quelque chose à cette fameuse Remington 22 ». Quand la fée de l'écriture "semble de bonne humeur ", tout avance bien : "Je m'assois pour écrire. La Remington semble de bonne humeur. Je tape comme un dingue. Ça crépite dans la nuit. Les phrases fusent à toute allure. Je ris » (82). C'est donc avec et de la machine, la "vieille complice » (161) que sort le résultat du livre qui boucle le récit quand le narrateur satisfait de son prodige présente le trio auteur, livre, machine. "Le roman me regarde là, sur la table, à côté de la vieille Remington, dans un gros classeur rouge. Il est dodu comme un dogue, mon roman. Ma seule chance » (163), voilà les mots qui closent le récit.

En sus de l'outil matériel de la machine, le livre se donne dans Comment faire l'amour avec un Nègre sans se fatiguer comme le fruit d'un grand investissement personnel. II est d'abord un objet qui se pense, se rêve au fur et à mesure qu'il s'écrit. Bien avant de passer à la machine, le texte mûrit dans la tête. À cette étape préliminaire, il est porté par des bouts de papiers, des notes, nourri des idées qu'inspirent le milieu, les gens croisés, les réflexions, les besoins personnels, etc. L'alliage donne ce que le long paragraphe du dixième chapitre du roman consacré à la Remington qualifie de «sorte de fourre-tout autobiographique où se retrouvent, pêle-mêle, début de roman, journal incomplet, rendez-vous manqués » (61).

Le livre donné à lire par Comment faire l'amour avec un Nègre sans se fatiguer se fait dans le temps comme durée et comme moment propice à l'acte d'écriture. Le pressentiment de Vieux, par exemple, que sa « nuit sera longue » (145) est confirmé quand il fait le compte et constate :

Je me suis enfermé depuis trois jours avec une caisse de bières Molson, trois bouteilles de vin, deux boîtes de spaghettis Ronzoni, cinq livres de pommes de terre et cette maudite Remington. J'ai affiché en bas, près de la sonnerie, un avertissement on ne peut plus clair: "NE DÉRANGEZ PAS LE GRAND ÉCRIVAIN, IL EST EN TRAIN D'ÉCRIRE SON ULTIME CHEF-D'OEUVRE. » Au bout de trois jours à taper sans arrêt, les petites lettres m'apparaissent irisées. [...] Les lettres capitales ressemblent plutôt à ces araignées poilues des tropiques. [...] Les consonnes n'arrêtent pas de forniquer et d'engendrer, là, sous mon nez. (147)

Au travers de ce passage, le narrateur laisse clairement entendre qu'écrire implique trimer, s'astreindre à un effort et à une discipline qui isolent l'individu. Un effort qui n'est pas par contre à l'abri des piétinements, des hésitations, des doutes, des incertitudes, des tentations d'abandonner. Le dialogue entre Bouba et Vieux révèle tout sous cet angle : «T'as pas l'air d'aller, Vieux ? [...] C'est à cause du bouquin ? Oui... C'est le vase. Je n'arrive à rien. - Tu devrais aller faire un tour. - Ça fait la dixième fois que tu me conseilles ça, Bouba. - Tu sais quoi, Vieux ? [...] Tu penses trop, c'est ça ton problème » (94). II ressort également de la suite du dialogue que si l'auteur peut être inspiré, il ne peut pas être aidé. C'est ainsi que Bouba qui voit son ami déconcerté et offre de l'aider si besoin en est se voit rétorquer : «Malheureusement, non, c'est le genre de truc qu'il faut faire soi-même »(94). La seule aide possible n'est pas de l'ordre de la création. Tout au long du chemin du travail d'écrire, aucune troisième main possible, seulement des divertissements tels que la musique, la beuverie, le sexe.

Profitons de la brèche qu'ouvre ce dernier élément du divertissement pour souligner encore le rôle de diversion que joue le thème de la sexualité dans le texte. L'acte sexuel, en dehors du volet des rapports sociaux qui informent le texte, n'a de valeur autre que celle dont tout élément de consommation est investi. La dimension stylistique du texte joue beaucoup sur ce plan. Faisons cas des constructions zeugmatiques suivantes : "Un peu en avant de moi, une fille sort de la librairie Hachette avec un Miller sous le bras et presque rien sur le corps » (54), "Miz Littérature est venue dans mon lit. J'ai déposé le livre au pied du lit, près de la bouteille de vin, avant de la renverser (Miz Littérature) » (44). Plutôt que d'être envisagés dans une relation de substitution, les thèmes du sexe et du livre devraient l'être dans celle de superposition, celui-là n'étant qu'au service de celui-ci. Vieux ne déclare-t-il pas: "Le sexe encore huilé. Mon corps parfumé de toutes les odeurs de Miz Sophisticated Lady. J'écris. Je suis heureux. Je le sais » (82) ? Le sexe se fait un aphrodisiaque pour l'écriture. Quand arrive le temps de faire le compte, Vieux peut soupirer :

Je vois, enfin le bout de ce bouquin de malheur. La Remington (ma vieille complice) semble avoir gardé la forme. Je n'aurai plus qu'à ajouter ce prologue. Tout bien compté, j'ai écrit ce roman en trente-six jours et dix-huit nuits, et j'ai utilisé trois rubans, quatre tubes à effacer, cinq cents feuilles (papier bon), trente bouteilles de vin, douze caisses de bières. Je tiens cette comptabilité dans un minuscule carnet 
Basabose, Philippe. " La représentation du livre dans

Comment faire l'amour avec un Nègre sans se fatiguer de Dany Laferrière » Nouvelle Revue Synergies Canada, N 13 (2020)

noir, cadeau de Miz Littérature. Je tape avec frénésie. La Remington jubile. Ça gicle de partout. Je tape. Je n'en peux plus. Je tape. J'en ai ma claque. J'achève. Je m'affale sur la table, à côté de la machine à écrire, la tête entre les bras. (160-161)

L'aboutissement du travail que nous avons tenté de décrire est un manuscrit qui a un titre - PARADIS DU DRAGUEUR NĖGRE (150) -, qui est divisé en chapitres, qui a un prologue, qui passe chez un éditeur, qui arrive dans des librairies, qui s'achète, qui trouve des lecteurs, qui se commente sur les plateaux de télévision, bref qui passe par tous les rouages de la « réification " ${ }^{6}$ (Jimenez 57) de l'objet esthétique. Comment, en effet, ne pas lire Comment faire l'amour avec Nègre sans se fatiguer comme un livre in the making, en train de s'écrire, un objet qui advient au fil de la construction du récit du roman et qui le domine comme une mise en abyme qui lui est consubstantielle?

\section{Des effets du livre}

Nous ne pourrions clore cette analyse du phénomène livre dans Comment faire l'amour avec un Nègre sans se fatiguer sous ses diverses facettes, notamment ses angles complémentaires de l'écriture et de la lecture, sans dire un mot sur ses effets multiformes. D'autant plus que le narrateur écrivain fixe des objectifs précis à son projet. Ce sont les classiques questions sartriennes du «pourquoi écrire? », "pour qui écrire ? » ramenées à l'échelle du roman en présence.

Le vœu formulé par Vieux est bien clair. II cherche à « [s]'en sortir » (148) et son effort est orienté vers cet effet quoiqu'il ne se fasse pas d'illusion sur la relativité, le coup de chance qui règle le succès :

II suffit d'avoir une bonne Remington, d'être sans le sou, sans éditeur, pour croire que l'ouvrage qu'on est en train d'écrire avec la violence de ses tripes est le chef-d'œuvre qui vous sortira du trou. Malheureusement, ce n'est jamais le cas. II faut autant de tripes pour faire un bon livre que pour en faire un mauvais. Quand on ne possède rien, on espère, au moins, le génie. Mais le génie a la gueule fine. II n'aime pas les démunis. Je suis nu comme un ver. Je ne pourrai jamais m'en sortir avec un manuscrit moyen. (148)

Pour se garantir le succès, Vieux est animé d'un grand esprit d'émulation et il se met la barre le plus haut possible. Le lecteur n'aurait pas tort de soutenir en effet que Vieux est obsédé de grands noms, qu'il puise de toutes les littératures, de toutes les époques, qu'il constitue en modèles mais aussi en émules, qu'il entend d'ailleurs surpasser. Les prétentions qu'affiche le dialogue suivant, entre Bouba et Vieux, militent bien en faveur d'un tel argument :

Donc, tu veux devenir le meilleur écrivain nègre ? - C'est ça. Meilleur que Dick Wright. - Meilleur que Chester Himes ? - Meilleur que Chester. - Meilleur que James Baldwin ? - Oh ! celui-là est un os dur. - Meilleur que Baldwin ou pas ? - Meilleur que Baldwin. Baldwin, joli nom, hein ? Avec Paradis du dragueur nègre, UN JEUNE ÉCRIVAIN NOIR DE MONTRÉAL VIENT D’ENVOYER JAMES BALDWIN SE RHABILLER. (95)

Vieux vivrait-il le rêve du vrai auteur (Dany Laferrière) qui, à ses premiers pas dans la littérature romanesque, n'aurait osé se mesurer aux grosses souches littéraires ? Le portrait substitutif compenserait bien la part de ce que l'humilité laferriérienne étoufferait. Nous ne pouvons ici priver le lecteur d'une anecdote qui peut en dire long sur le rapport que Laferrière a une fois dit entretenir avec les grandissimes de la littérature. L'auteur de Comment faire l'amour avec un Nègre sans se fatiguer participait, en mars 2009, comme invité d'honneur, à une conférence dédiée à Aimé Césaire et organisée à l'Université McMaster. Alors qu'il venait de finir son allocution inaugurale, un participant lui demanda s'il avait rencontré Aimé Césaire. Comme si Laferrière avait médité la question depuis longtemps, il répliqua que non il n'avait jamais rencontré Aimé Césaire, qu'on ne rencontre pas son Dieu. Les dieux de la vraie vie, c'est-à-dire les auteurs de prédilection et de référence de Dany Laferrière, sont les familiers, les rivaux non imbattables de l'espace romanesque.

Suivant le degré de l'effet qu'un livre suscite chez son public, il peut être différemment qualifié. Le texte aligne, entre autres qualificatifs, les mots suivants : " bon » (148), " très bon » (131), " chef-d'œuvre » (148), « moyen (148), « mauvais » (96), « de la merde » (118), etc. Évaluant son livre à l'état achevé, Vieux décrit fièrement : " Mon livre est placé entre Moravia et Green. En bonne compagnie. Ce livre, tranquillement assis, cette couverture jaune et rouge, cet effet jazz, c'est MOI. MOI TOUT ENTIER. Je suis ces cent soixante pages bien tassées » (149). Se tailler une place entre l'auteur Alberto Moravia et Julien Green, auteurs qui s'ajoutent 
Basabose, Philippe. " La représentation du livre dans Comment faire l'amour avec un Nègre sans se fatiguer de Dany Laferrière » Nouvelle Revue Synergies Canada, N 13 (2020)

à la série de bien d'autres grands noms littéraires, revient à promettre le nouvel ouvrage à une grande destinée, celle des « grands livres » dans le sens de Patrick Chamoiseau qui écrit :

Le grand livre [...] n'est d'aucun âge ou d'aucune histoire ; [...] souple, ouvert, riche d'une ambiguïté transversale, il lui arrive de résonner dans les urgences, dans les blessures, les âges, les histoires. [...] Ses couches d'ombres sont nombreuses, et chacune, dans ses ambiguïtés, porte germe de mille réveils possibles » (30-31)

La critique journalistique rivalise de superlatifs ${ }^{7}$ : «Le supplément littéraire du samedi de La Presse titrait (parlant de moi) : "Le NOUVEAU PRODIGE » (152). Paradis du dragueur Nègre fait la fierté de son auteur et il le tirera peut-être de sa situation de subalterne, de démuni, d'anonyme, comme il en nourrit l'ambition dès sa conception.

À côté de ces visées pratiques, matérialistes, le livre est inscrit dans Comment faire l'amour avec un Nègre sans se fatiguer en tant qu'il est appelé à correspondre, à répondre aux différents " horizons d'attente ${ }^{8}$ de ses divers lecteurs. Le livre peut être bienfaisant, curatif même ou, au contraire, nuisible. Pour le premier pôle, nous avons déjà évoqué le livre comme outil de la cure psychanalytique. Pour le deuxième, le roman ne cache pas que le livre peut avoir, comme la chanson ou le cinéma, un effet destructif. C'est par le biais du regrettable constat de Vieux que la stratégie typographique (les majuscules) renforce :

ET DIRE QU'ON ENVOIE CES FILLES DANS UNE INSTITUTION SÉRIEUSE (MCGILL) POUR APPRENDRE LA CLARTÉ, L'ANALYSE ET LE DOUTE SCIENTIFIQUE. ELLES SONT TELLEMENT INFECTÉES PAR LA PROPAGANDE JUDÉO-CHRÉTIENNE QUE DĖS QU'ELLES PARLENT À UN NÈGRE, ELLES SE METTENT À PENSER EN PRIMITIVES. POUR ELLES, UN NĖGRE EST TROP NAÏF POUR MENTIR. C'EST PAS LEUR FAUTE, IL Y A EU, AUPARAVANT, LA BIBLE, ROUSSEAU, LE BLUES, HOLLYWOOD, ETC. (114)

Comme les parties précédentes de notre analyse, il s'avère que celle-ci, qui est centrée sur le volet de la réception de l'œuvre - celle enchâssée dans le roman - colle bien avec sa thématique. Aussi bien dans sa réponse aux attentes qu'en nourrissait le narrateur écrivain que dans la façon dont le public acquiert et accueille l'objet-livre et en partage le contenu et les enjeux.

\section{Conclusion}

Qu'on l'envisage du point de vue de son déploiement topographique, de son architecture narrative ou de la place que son intrigue fait au circuit complet de la conception du livre jusqu'à sa réception et aux effets de celle-ci, le roman Comment faire l'amour avec un Nègre sans se fatiguer est une représentation de l'objet-livre. En sus de ces diverses étapes qui font advenir le livre ou concourent à son achèvement (dans la lecture), Dany Laferrière a fait de son premier roman le reflet de sa culture littéraire, rendant ainsi hommage aux auteurs qui ont façonné son être intellectuel et s'assurant en même temps, et par ce fait même, une sorte d'auto-légitimation dans l'accession au champ de l'écriture. La présence nettement marquée du motif du livre à travers toutes ces facettes est à situer dans la logique de l'autoréflexivité que Jonathan Culler reconnaît à la littérature quand il souligne que "la possibilité de lire un texte littéraire comme une réflexion sur sa propre nature et sur celle de la littérature fait de la littérature un discours autoréflexif, un discours qui, implicitement [...] raconte quelque chose d'intéressant sur sa propre activité significative » (39). II serait intéressant d'étudier à quel degré cette poétique qui constitue l'angle d'attaque sous lequel Laferrière réalise son entrée dans l'art d'écrire irradie de Comment faire l'amour avec un Nègre sans se fatiguer vers son œuvre subséquente.

\section{Notes}

${ }^{1}$ Pour la suite des références, nous n'indiquerons que le numéro de la page pour les renvois au texte de Comment faire l'amour avec un Nègre sans se fatiguer.

${ }^{2}$ C'est nous qui soulignons.

${ }^{3}$ Rappelons que le titre de l'avant-dernier chapitre d'un autre roman de Dany Laferrière, Je suis un écrivain japonais, fait aussi de la place à Borges : « Je ne suis pas Borges et M. Tanizaki n'est pas le bon » (255). 
Basabose, Philippe. " La représentation du livre dans Comment faire l'amour avec un Nègre sans se fatiguer de Dany Laferrière " Nouvelle Revue Synergies Canada, N 13 (2020)

${ }^{4}$ La question identitaire dans l'œuvre de Dany Laferrière a fait l'objet de beaucoup d'études et nous ferons ici référence, à titre indicatif, à celles de Bonnie Thomas (2010), Jimmy Thibeault (2011), Arthur Mukenge et Viviane Kayumba (2018).

${ }^{5}$ En disant méta-inscrites, nous avons à l'esprit le passage où le narrateur-auteur discute ses rapports avec son personnage avec une journaliste sur le plateau de Radio Canada (154-157).

${ }^{6}$ L'analyste de l'œuvre de Theodor Adorno faisait remarquer : « L'erreur consiste à croire que la sphère de l'esthétique reste imperméable aux influences extérieures, que l'acte de "création » artistique échappe à la réification, aux ravages de l'industrie culturelle et au dirigisme de l'idéologie dominante » (Jimenez 57).

${ }^{7}$ Levons ici toute équivoque en précisant qu'il s'agit de critiques du roman fictif de Vieux.

${ }^{8}$ Nous saisissons le mot dans le sens de Hans Robert Jauss quand il écrit : « Le lecteur ne peut 'faire parler' un texte, c'est-à-dire concrétiser en une signification actuelle le sens potentiel de l'œuvre, qu'autant qu'il insère sa précompréhension du monde et de la vie dans le cadre de référence littéraire impliqué par le texte. Cette compréhension du lecteur inclut les attentes concrètes correspondant à l'horizon de ses intérêts, désirs, besoins et expériences tels qu'ils sont déterminés par la société et la classe à laquelle il appartient aussi bien que par son histoire individuelle » (259).

\section{Bibliographie}

Boivin, Aurélien. "Comment faire l'amour avec un Nègre sans se fatiguer ou une dénonciation du racisme à travers la baise ». Québec français, no. 131, 2003, pp. 94-97.

Chamoiseau, Patrick. Écrire en pays dominé. Gallimard, 1997.

Culler, Jonathan. "La littérarité ». Théorie littéraire, édité par Marc Angenot et al., Presses Universitaires de France, 1989, pp. 31-43.

Genette, Gérard. Palimpsestes. La littérature au second degré. Seuil, 1982.

Hertel, François. Du métalangage. Éditions de la Diaspora Française, 1968.

Jauss, Hans Robert. Pour une théorie de la réception. Gallimard, 1978.

Jimenez, Marc. Adorno : art, idéologie et théorie de l'art. Union Générale d’Éditions, 1973.

Laferrière, Dany. Comment faire l'amour avec un Nègre sans se fatiguer. Typo, 2002.

---. L'énigme du retour. Audiolib, 2010.

---. Je suis un écrivain japonais. Boréal, 2008.

Lamontagne, André. « 'On ne naît pas Nègre, on le devient' : La représentation de l'autre dans Comment faire l'amour avec un Nègre sans se fatiguer de Dany Laferrière ». Québec Studies, vol. 23, 1997, pp. 2942.

---. Le roman québécois contemporain : les voix sous les mots. Fides, 2004.

Lessard, Alain. «Borges : l'érudition merveilleuse. Entrevue avec Dany Laferrière ». Nuit Blanche, no. 120, 2010, pp. 34-38.

Mukenge, Arthur et Viviane Kayumba. "Le style de Dany Laferrière dans la construction d'une identité pérégrine. Cas de : Le cri des oiseaux et Le goût des jeunes filles ». Les Cahiers du GRELCEF, no. 10, 2018, pp. 125-142.

Papini, Giovanni. Miroir qui fuit. Panama, 2006. 
Basabose, Philippe. " La représentation du livre dans Comment faire l'amour avec un Nègre sans se fatiguer de Dany Laferrière " Nouvelle Revue Synergies Canada, N 13 (2020)

Saint-Martin, Lori. « Une oppression peut en cacher une autre ». Voix et Images, vol. 36, no. 2 (107), hiver 2011, pp. 54-67.

Thibeault, Jimmy. "'Je suis un individu'. Le projet d'individualité dans l'œuvre romanesque de Dany Laferrière ». Voix et Images, vol. 36, no. 2, 2011, pp. 25-40.

Thomas, Bonnie. « Migrations of identity in Dany Laferrière's Une autobiographie américaine ». Australian Journal of French Studies, vol. 47, no. 3, 2010, pp. 266-276.

Verstraet, Charly. «Trick or Treat : Déconstruction d'une transgression chez Dany Laferrière ». Nouvelles Études Francophones, vol. 31, no. 1, 2016, pp. 132-147. 